\title{
A Research on Innovative Design Method of Modern Furniture Based on Without Thought
}

\author{
Wenyin Dong ${ }^{1, a}$, Jiamin Huang ${ }^{2, b}$ Xiangdong Dai ${ }^{3, c}$ \\ ${ }^{1}$ Forestry College, Sichuan Agricultural University, Chengdu, China \\ ${ }^{2}$ Forestry College, Sichuan Agricultural University, Chengdu, China \\ ${ }^{3}$ Central South University of Forestry and Technology, Changsha, China \\ adoreen0656@126.com, b474752253@qq.com, ‘642344070@qq.com
}

Keywords: Without Thought, Modern furniture, Innovative design

Abstract. In the age of the flush creative products, products with the idea of Without Thought can always agree with users' preference, which makes them occupy a good market. By studying product design method based on Without Thought, this thesis will try to explore the innovative design method from different fields of disciplines.

\section{Introduction}

In order to enhance "different" characteristics of products, modern designers strive for innovation through multifunctional additional combination and diversified modeling and colors. However, at the same time, it ignores users' irrational preference to new products and increases their "burden of adaptation". But MUJI, which is based on the concept of Without Thought, is widely favored by Japanese and even people all around the world. Without Thought put forward by Naoto Fukasawa is based on the height of "people oriented", which endows products with emotions and makes them not only satisfy the use function, but also stimulate users' positive emotion. In this way, the independently products and user emotions are connected by users' "use behavior", thus producing resonation, satisfying the consumers' potential needs and effectively eliminating the misunderstanding between people and products.

\section{Without Thought}

"Without Thought" is a design concept put forward by Naoto Fukasawa according to Jung Carl Gustav's "Collective Unconsciousness", which means "transforming unconscious behavior into visible things". It is not a new design method, but instead, it is a guiding concept aimed at solving objectively existing problems; it is also not a rootless presentation of originality, but it is a combination of art and technology oriented at "people first".

\section{Application Principle of Without Thought}

The world we know is not simply an objectively existing physical world, but it is an integrated environment involving humans' multiple perceptual knowledge.

According to physiological analysis, the brain's nerve cell only has two states: excitement and no excitement (i.e. restraint). If the total of signals entering the brain from the dendrite's synapsis surpasses a certain threshold value, it will stimulate the nerve cell to enter the state of excitement. On the contrary, the nerve cell will not be excited. Here the total of signals refers to the overall stimulation that the modern furniture offers to users. If the energy of such stimulation can surpass the threshold value arousing people's excitement (fragments of memory or experience of usage) based on the original "subliminal", the modern furniture based on Without Thought (hereinafter referred to as "Without Thought furniture" can reach the expected design effect.

Based on psychological analysis, Jung Carl Gustav "defines unconsciousness as the total of all psychological phenomena that have been realized. These psychological contents can be correctly 
referred to as 'subliminal'. That is, supposing that any psychological content must have a certain energy value so as to be realized, the lower the energy value of certain consciousness content is, the more easily it will disappear in the subliminal." In designs, the first thing that Naoto Fukasawa thought of is to materialize the good things that everyone can feel but haven't been realized (collective unconsciousness). That is to say, increase the energy value of certain "subliminal" consciousness content, making its energy enough to step over the "subliminal" limits. In a manner of speaking, the information presented by Without Thought furniture is like the presupposed incentive condition, and its stimulation to users can be received by humans' sensory organs, and in this way, users' multiple subject consciousness will be aroused, the unordered trivial fragments will be connected, and then through the progressing and transformation from the perceptual layer to the consciousness layer, its function gradually produce effect on users' behavior or thoughts.

\section{An Innovative Design Method with Divergent Thinking Based on Without Thought - Increasing the "subliminal" Energy Value}

The design of Without Thought furniture needs a systematic and scientific method, such as psychology, behavioristics and ergonomics. By looking into some cases, try to find out the universal development trend of things, summarize relevant laws and expand details that the public want but haven't been realized, and then deal with them in product design.

The author believes that if quantitative analysis is carried out on the design of modern furniture, the energy of modern furniture can be defined as the total of three parts. That is, basic threshold, increased threshold and additional threshold. Under the condition of basic threshold, by increasing threshold, it is likely to surpass the "subliminal" energy value, and the additional threshold makes the whole product more accordant to the objective law of "principle".

Basic threshold-satisfy users' demand. From the perspective of users, Naoto Fukasawa explains that product design is neither a pure finding of needs nor a blind creation of needs; instead, it presents the things existing in people's subconsciousness in a beautiful form and them meet ${ }^{[1]}$. The user's demand is not only the explicit demand for the product. Take cost performance for example. Actually, the potential need hiding inside users' subconsciousness is relatively large, which is usually difficult for users to put forward and even perceive. Potential needs can usually satisfy users' demand for life quality.

For example, the tray table lamp created by Naoto Fukasawa. When people arrive home after a tiring day, they can naturally put keys into the tray under the table lamp, when the lamp will light automatically. In this way, it can also guide peoples' correct habit of storage. This work originates from reflection on life details, which not only makes the product's emotions actively stimulate users' emotion of need, but also let users experience the sense of joy gained through the response of their needs and thinking in their subconsciousness, thus realizing the optimal coordination of the dominant function and the potential function in Without Thought furniture.

Increased threshold-Endow products with symbolic meaning. Product modality is a symbolic system, which consists of symbolic form (signifier) and symbolic meaning (signified). According to the combination methods of different shapes, colors and textures, they are ranked into rich codes. Designers use multiple ways like analogy, association, metaphor and simile to establish the connection between products and users. Then they are accepted by people through people's sensory systems like vision, hearing and feeling and further decoded by people through cognitive thinking activities.

Image symbol. Graphical symbol expresses itself through "similar image" simulation or existing facts of likeness by means of things with existing meaning. This is an original significance expressing method, which is direct and clear and of high "readability" ${ }^{[2]}$. Simulation and bionics - the most basic way of applying elements in life to design form through signifying, under the premise of ergonomics, extract certain common pictographic or biological features to carry out creative structure and recombination so as to design furniture which is quite like certain shapes or accordant to certain biological principles and features. This is an important technique of innovative design of furniture ${ }^{[3]}$. 
For example, flower chair. The designer abstracts the abstract and simple geometric form of lily, according to principles of ergonomics and people's effective size, consciously connect the "bearing effect" of the center of petal and the chair. In this way, under the influence of subconsciousness, users will sit down naturally.

Directional symbol. Directional symbol makes use of the symbolic forms and the "inevitable and essential" physical connection of the significance to be expressed, and constitutes reference function based on the perception from cause to effect so as to make people know the significance ${ }^{[3]}$. For example, the cord pulling CD player created by Naoto Fukasawa. Its appearance is like a wall-mounted fan, and when the cord is pulled, the CD player runs like air wings. In this way, instead of wind, touching music comes out. Naoto Fukasawa skillfully grasps the consciousness core of people's common cognition towards everything to arouse people's connective cognition memory. The design of the cord and simulated fan makes people pull the cord unconsciously, when the cord of the CD player can be viewed at the symbolic manifestation of directional symbols.

Symbolic symbols. The form and the symbolic objects of symbolic symbols don't have connection in similarity or cause and effect. Their representation is only established on certain conventional significance in society ${ }^{[4]}$, and people express their spiritual content that can't be expressed with words by means of these symbolic symbols. Shi Dayu, gentleman of bamboos, shows special love for bamboo wares. At the same time, he is also a person like bamboo-tough and straight after going through so many hardships. Either in Chinese industrial design or literary ideological trend, bamboo exists in public mind with its fresh and modest image. Relaxing Chair-A chair created by Mr. Shi Dayu, has a harmonious curving and straight modeling, which is like circle in a square seen from the side. It completely adopts the disposition of lines and radians, simple but flexible. The whole product motivates the public's common cognitive competence from the essential materials to potential emotions from the inside out, in which it reflects the natural and real symbol of bamboo and has dense regionalism of China.

Additional threshold-Harmony between human and machine. Naoto Fukasawa mentioned, "the relationship between objects and environment is more important than the object itself. The object is a part of harmony. I start to stop the simply interesting visualization and begin to consider relationship among objects. As for design, it is necessary to abstract some elements matched with environment from various elements in your living environment." Thus it can be seen that the product is not simply the standard measurement of single dimensions like beauty, utility, easiness of use, humanization, environmental protection and technical innovation, but instead, it is a synthesis which maintains good relationship with users and environment. It will appear and disappear at proper time; the product has no trace of "deliberate" design, bringing us natural experience beyond visual interest ${ }^{[1]}$.

For example, Fukazawa Naoto's printer with wastebasket, in order to select the most satisfactory result, there will be redundant paper printed out, and people's habitual action is to throw away the rest of the other. However, the wastebasket printer achieves the effective unify of man-machine environment, for the bottom of the printer is directly designed as a paper basket, suggesting and guiding people to throw the waste paper into the rubbish bin, so that the redundant paper can be recycled. This design not only saves space, but also protects the environment. Therefore, in the design process, we should pay attention to the details of contradictions between people and the environment, and solve the problem by changing the function or shape of Without Thought furniture, so that the Without Thought furniture has transparency that it's form can disappear in the environment, but it's function inevitably exists in the environment. It is emphasized that the transparency is not only purely visual continuity, but an attempt that combines modern high-tech with region of natural materials, and it considers the "people - machine - environment" as a whole, to achieve harmony. The design conforms to even more order the user's habits.

\section{Conclusions}

With the arrival of the era of perceptual consumption, the value of commodities added emotional factors has became a new benchmark to determine the quality. Considering the innovational design is 
not limited to the artistic field. Combining with the physiology, psychology, semiotics, etc for the modern furniture design system can provide a new idea or method for design, and greatly enrich the contents of the furniture innovational design research.

\section{Acknowledgements}

This work was financially supported by the major project of humanities and social sciences of education department of Sichuan province (16SA0006), the project of Undergraduate Scientific Research Interest of Sichuan Agricultural University, the research program of Allied Social Science Association of Sichuan Agricultural University (2013YB01).

\section{References}

[1] Y. N. Huang, Back to the origin of product design -- Analysis of Fukazawa Naohito's idea and design practice. Decoration Vol. 11 (2008), p.112-114

[2] F. Hu, The research of art design's symbol, meaning and application. Wuhan University of Technology (2002)

[3] J. Wang, k. li, The analyses of furniture design and Semiotics. Popular literature Vol.17 (2002), p.93-94

[4] J. H. Yu, The research on furniture form based on the theory of semiotics. Nanjing Forestry University (2009) 